\title{
Caso 3/2007 - Insuficiência Cardíaca e Bloqueio Atrioventricular da Condução do Estímulo em Mulher de 59 Anos Portadora de Sarcoidose
}

Case 3/2007 - Heart Failure and Conduction Disturbance in a 59 Year-Old Female with Sarcoidosis

Fábio de Moraes Medeiros, Luis Fernando B. C. Seguro, Jussara Bianchi Castelli

Instituto do Coração do Hospital das Clínicas - FMUSP, São Paulo, SP

Paciente de 59 anos de idade, natural de Passagem (MG) e procedente de São Paulo, sabia ser portadora de sarcoidose hepática e pulmonar e foi encaminhada para consulta no hospital (18/10/2001) em razão de queixa de aceleração no coração há dez meses, além de fadiga e cansaço quando caminhava e do surgimento de edema. Houve constatação de cardiomegalia em radiografia de tórax. Fazia uso de prednisona, espironolactona e furosemida.

A biópsia hepática (29/11/2000) revelou bloqueio do efluxo venoso e hepatite granulomatosa (granulomas epitelióides com células gigantes e necrose focal).

A paciente havia recebido inicialmente tratamento para tuberculose pulmonar. Apresentava síndrome de Cushing pelo uso prolongado de prednisona.

O histórico familiar incluía pai falecido aos 58 anos de idade por asma brônquica; mãe falecida aos 58 anos em conseqüência de diabete melito. Um irmão da paciente havia falecido aos 54 anos em razão de enfisema pulmonar e asma, e outro havia falecido aos 28 anos por suicídio.

O exame físico (18/10/2001) revelou peso de $96 \mathrm{~kg}$, altura de $157 \mathrm{~cm}$, pulso com 100 batimentos por minuto, com presença de extra-sístoles, pressão arterial 120x80 mmHg. A semiologia pulmonar não revelou alterações. O exame do coração mostrou ritmo cardíaco regular com presença de extra-sístoles freqüentes. O abdome era globoso e de difícil palpação.

O eletrocardiograma (15/10/2001) revelou extra-sístoles supraventriculares e ventriculares, bloqueio da divisão ânterosuperior do ramo esquerdo e bloqueio do ramo direito do feixe de His (fig. 1).

A radiografia do tórax mostrava aumento da área cardíaca $++/ 4$, aumento da trama vasobrônquica em bases pulmonares e aorta alongada.

\section{Palavras-chave}

Débito cardíaco, bloqueio cardíaco, cardiomegalia, sarcoidose.

Editor da Seção: Alfredo José Mansur (ajmansur@incor.usp.br) Editores Associados: Desidério Favarato (dclfavarato@incor.usp.br) Vera Demarchi Aiello (anpvera@incor.usp.br)

Correspondência: Vera Demarchi Aiello •

InCor - Av. Dr. Enéas de Carvalho Aguiar, 44 - 05403-000 - São Paulo, SP E-mail: anpvera@incor.usp.br
A tomografia de tórax (12/3/2003) revelou espessamento difuso das paredes brônquicas, linfonodo diafragmático menor que $1 \mathrm{~cm}$ à direita e aumento de número dos linfonodos axilares, todos com diâmetros menores que $1 \mathrm{~cm}$. Na tomografia abdominal havia massa supra-ísquio-retal com dimensões de $15 \mathrm{~cm} \times 8 \mathrm{~cm} \times 6,5 \mathrm{~cm}$.

O exame ultra-sonográfico do abdome evidenciou hepatomegalia, nódulos hepáticos, sinais de hipertensão portal e de hepatopatia crônica.

Os exames laboratoriais (15/10/2001) mostravam: hemoglobina 12,6 g/dl, hematócrito 38\%, eritrócitos 4200000 por $\mathrm{mm}^{3}$, leucócitos 8700 por mm³, colesterolemia $364 \mathrm{mg} /$ dl, triglicérides $91 \mathrm{mg} / \mathrm{dl}$, creatinina 0,9 mg/dl, glicemia 119 $\mathrm{mg} / \mathrm{dl}$. As pesquisas de anticorpos antimitocôndrias, anticélula parietal, antimúsculo liso renal e gástrico, antimicrossoma de fígado e rim, anticorpo anticitosol hepático foram negativas. A adenosina deaminase foi de $8,3 \mathrm{U} / \mathrm{l}$ no liquido ascítico. A pesquisa de bacilos álcool-ácido resistentes no lavado brônquico foi negativa; fosfatase alcalina 500 U/l, ALT 135 U/l, AST $96 \mathrm{U} / \mathrm{l}$, gama GT $1165 \mathrm{U} / \mathrm{l}$, desidrogenase láctica $499 \mathrm{U} / \mathrm{l}$; colesterol 258 mg/dl (HDL-C 100 mg/dl, LDL-C 144 mg/dl) e triglicérides $72 \mathrm{mg} / \mathrm{dl}$. O coagulograma era normal.

Foram acrescentados atenolol $50 \mathrm{mg}$, sinvastatina $20 \mathrm{mg}$ e enalapril $5 \mathrm{mg}$ ao tratamento que a paciente vinha recebendo.

O ecocardiograma (1/2/2002) revelou espessura do septo interventricular $10 \mathrm{~mm}$, de parede posterior do ventrículo esquerdo $9 \mathrm{~mm}$, diâmetros de ventrículo esquerdo 51/32 (diastólico/sistólico), fração de ejeção do ventrículo esquerdo $75 \%$. As valvas mitral e aórtica exibiram espessamento. O ventrículo e átrio direitos e o pericárdio foram considerados normais. O exame com Doppler demonstrou insuficiência mitral de grau discreto.

A ressonância magnética $(2 / 2 / 2002)$ revelou disfunção discreta do ventrículo esquerdo, com realce tardio dos ventrículos direito e esquerdo, compatível com cardiomiopatia de etiologia não-isquêmica.

A reavaliação clínica na evolução (7/2/2002) evidenciou melhora da palpitação. Não houve mudança significativa no seguimento (2/10/2002, 6/6/2002). A partir desta última avaliação, recebeu a recomendação de ater-se ao seguimento pneumológico que já fazia anteriormente.

Foi suspenso o tratamento com prednisona e iniciado o tratamento imunossupressor.

Depois de dois meses (8/8/2002), retornou ao hospital com queixa de episódios de pré-síncope que se iniciaram havia 30 


\section{Correlação Anatomoclínica}

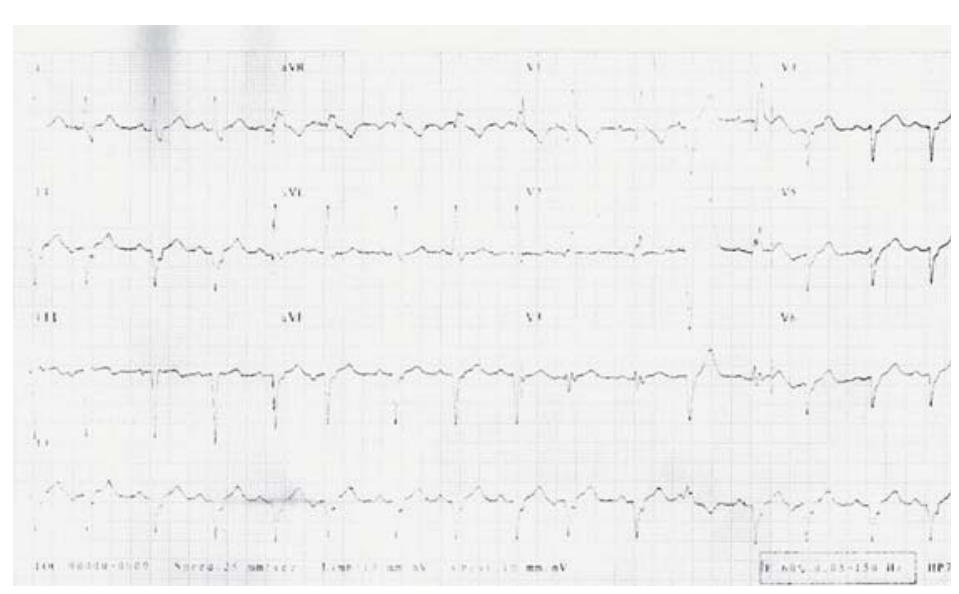

Fig. 1 - ECG - Extra-sístoles supraventriculares e ventriculares; bloqueio do ramo direito e da divisão ântero-superior do ramo esquerdo.

dias. Informou que em atendimento médico de emergência foi verificada pressão arterial $60 \times 40 \mathrm{mmHg}$, e no exame clínico a pressão arterial foi 130x70 mmHg em decúbito dorsal horizontal, e 110x60 mmHg em posição sentada. Foi feito o diagnóstico de hipotensão postural, suspenso o uso de atenolol e a paciente foi reencaminhada para nova avaliação.

O eletrocardiograma (28/8/2003) mostrou ritmo sinusal com períodos de taquicardia atrial, extra-sístoles supraventriculares, extra-sístoles ventriculares polimórficas, bloqueio divisional ântero-superior do ramo esquerdo e bloqueio do ramo direito do feixe de His (fig. 2).

Dois anos após (21/7/2005) apresentou episódio de présincope e foi constatada bradicardia, com 40 batimentos por minuto. O eletrocardiograma revelou bloqueio atrioventricular de $2^{\circ}$ grau tipo II de Mobitz. A paciente foi encaminhada para tratamento neste hospital.

O exame físico revelou paciente consciente, eupnéica, afebril, corada. A freqüência cardíaca foi de 40 batimentos por minuto, a pressão arterial foi de $110 \times 70 \mathrm{mmHg}$, a freqüência respiratória de 16 incursões por minuto. O exame dos pulmões não revelou alteração semiológica. O exame do coração revelou bulhas rítmicas, normofonéticas, sem sopros. O exame do abdome evidenciou ascite volumosa, com circulação colateral visível. Havia edema $++/ 4$ de membros inferiores.

A avaliação laboratorial é apresentada na tabela 1.

Foi implantado marcapasso provisório (21/7/2005) pela veia jugular direita; houve perda de captura e foi repassado em $23 / 7 / 2005$.

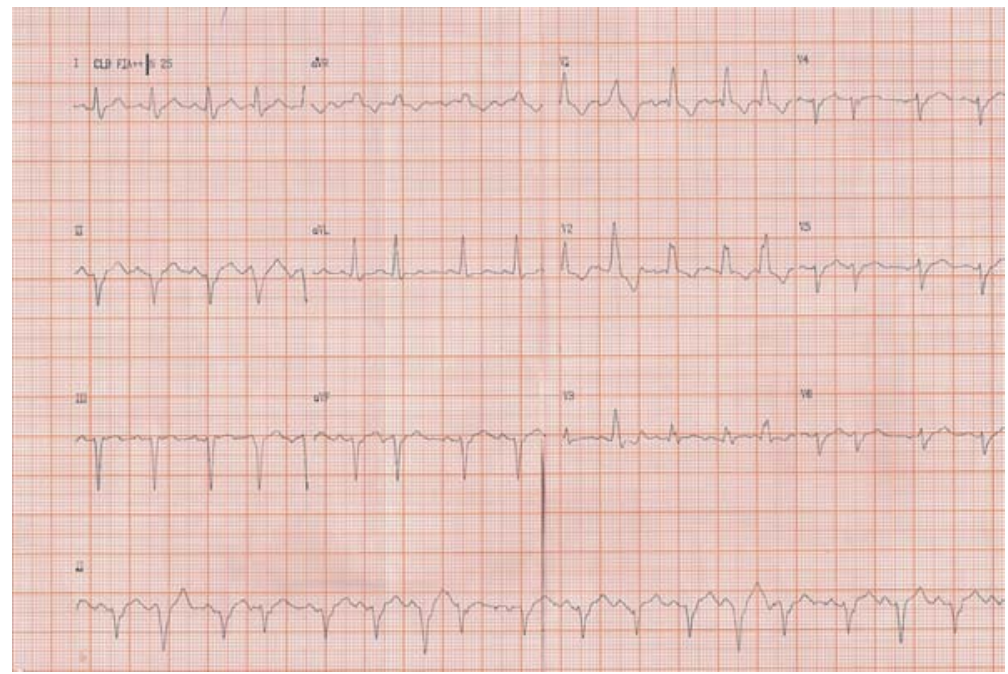

Fig. 2 - ECG - episódio de taquicardia atrial; extra-sístoles supraventriculares e ventriculares; bloqueio do ramo direito e da divisão ântero-superior do ramo esquerdo. 
Tabela 1 - Evolução laboratorial

\begin{tabular}{|c|c|c|c|c|c|c|}
\hline & 21 jul. & 25 jul. & 30 jul. & 31 jul. & 10 ago. & 2 ago. \\
\hline Hemoglobina (g/dl) & 10,5 & 11,3 & 12,5 & 13,4 & & 11,3 \\
\hline Hematócrito (\%) & 33 & 34 & & & & \\
\hline Leucócitos/mm³ & 3.600 & 3.700 & 18.500 & 16.100 & & 11.700 \\
\hline Plaquetas $/ \mathrm{mm}^{3}$ & 85.000 & 71.000 & 125.000 & & & 91.000 \\
\hline Uréia (mg/dl) & 34 & 37 & 91 & 129 & & 187 \\
\hline Creatinina (mg/dl) & 1 & 0,9 & 2,3 & 3,7 & 4,1 & 3,8 \\
\hline Sódio (mEq/l) & 140 & & 139 & 139 & 138 & 141 \\
\hline Potássio (mEq/l) & 4,2 & & 3,9 & 5,2 & 5 & 4,9 \\
\hline \multicolumn{7}{|l|}{ Bilirrubinas } \\
\hline Totais (mg/dl) & 1,46 & & & & 9,95 & \\
\hline Direta (mg/dl) & 0,5 & & & & 7,32 & \\
\hline Indireta (mg/dl) & 0,96 & & & & 2,63 & \\
\hline TGO/ALP (U/I) & & & & & 548 & \\
\hline TGP/AST (U/I) & & & & & 337 & \\
\hline Gama-GT (U/I) & & & & & 128 & \\
\hline Fosfatase alcalina (U/I) & & & & & 187 & \\
\hline Albumina (g/dl) & 3,5 & & & & & \\
\hline Cálcio (mg/dl) & 1,3 & & & & & \\
\hline Fósforo (mg/dl) & 2,6 & & & & & \\
\hline
\end{tabular}

O ecocardiograma (22/7/2005) revelou átrio esquerdo $49 \mathrm{~mm}$, aorta $29 \mathrm{~mm}$, ventrículo esquerdo $52 \mathrm{~mm} / 38 \mathrm{~mm}$ (diástole/sístole), espessura de septo interventricular e de parede posterior de ventrículo esquerdo $10 \mathrm{~mm}$, ventrículo direito 26 $\mathrm{mm}$. Havia aumento moderado do átrio esquerdo. O átrio e o ventrículo direito foram considerados normais. A pressão sistólica em ventrículo direito foi estimada em $44 \mathrm{mmHg}$. Havia discreto derrame pericárdico e o cabo do marcapasso estava posicionado em câmaras cardíacas direitas.

A cintilografia cardíaca com gálio 67 (28/7/2005) mostrou mínima hiperconcentração do radiofármaco em projeção do 1/3 médio do pulmão esquerdo. O estudo foi considerado negativo para processo inflamatório cardíaco em atividade e duvidoso para processo inflamatório pulmonar.

Foi implantado marcapasso provisório (10/8/2005) pela veia basílica esquerda.

Houve oscilação da captura do marcapasso provisório. Como não havia repercussão hemodinâmica, optou-se por conduta expectante antes do implante do marcapasso definitivo que havia sido planejado.

$\mathrm{Na}$ evolução, a paciente apresentou temperatura $38,5^{\circ} \mathrm{C}$, hiperemia e eliminação de secreção purulenta no local de implante do marcapasso. Foram colhidos novos exames laboratoriais (tab. 1), incluindo hemoculturas, e foi recomendado o tratamento com vancomicina e ciprofloxacina.

As hemoculturas revelaram crescimento de cocos Grampositivos (10/8/2005).
Três dias após apresentou adinamia, depois rebaixamento do nível de consciência. O exame físico revelou pressão arterial $110 \times 70 \mathrm{mmHg}$, freqüência cardíaca 89 batimentos por minuto. A avaliação laboratorial revelou deterioração da função renal (tab. 1). Instalou-se hipotensão arterial (pressão arterial 80x50 mmHg). Foi necessária intubação orotraqueal para apoio respiratório associando-se tratamento com dopamina.

Staphylococcus aureus sensível à oxacilina foi isolado na hemocultura.

Posteriormente, a dopamina foi substituída pela noradrenalina (4/8/2005).

A condição clínica da paciente deteriorou-se progressivamente, e ela veio a sofrer parada cardiorrespiratória, reanimada depois de 20 minutos. Uma hora depois, sofreu nova parada cardiorrespiratória, não responsiva às manobras de reanimação, e faleceu (4/8/2005).

\section{Aspectos clínicos}

O caso em questão refere-se a uma mulher de 59 anos de idade com diagnóstico de sarcoidose hepática e pulmonar que passou a apresentar sintomas de "aceleração" no coração, cansaço e edema.

A sarcoidose é uma doença sistêmica de etiologia desconhecida, caraterizada pela formação de granulomas não-caseosos em qualquer órgão, causando desarranjo de suas estruturas teciduais. A apresentação mais freqüente da 


\section{Correlação Anatomoclínica}

sarcoidose é a de um adulto jovem com linfadenopatia hilar bilateral, infiltrado pulmonar e lesões de pele ou olhos. Todavia, em análise de autópsia, encontram-se granulomas distribuídos difusamente, mesmo em pacientes com doença localizada ${ }^{1}$.

O acometimento do trato respiratório ocorre em quase todos os casos de sarcoidose em algum momento da evolução ${ }^{2}$. Em geral, apresenta-se como uma doença intersticial, envolvendo alvéolos, pequenos vasos sangüíneos e bronquíolos. Os principais sintomas são dispnéia e tosse seca.

A presença de linfonodomegalia intratorácica e periférica é muito característica da sarcoidose. Em 90\% dos indivíduos evidencia-se aumento de linfonodos hilares na radiografia de tórax ${ }^{3}$. A adenopatia periférica, embora muito comum, pode passar despercebida. Acomete principalmente gânglios cervicais, axilares e inguinais, sendo geralmente indolores e não-aderentes. Em alguns casos podem-se encontrar grandes nódulos, únicos ou múltiplos, passíveis de serem confundidos com tumores.

O acometimento hepático é também bastante freqüente, podendo-se encontrar granulomas em até $70 \%$ das biópsias hepáticas realizadas. No entanto, a presença de disfunção hepática é bastante rara. A apresentação mais comum, ocorrendo em um terço dos casos, é a de hepatomegalia acompanhada de aumento de enzimas canaliculares, com aumento mínimo de transaminases (padrão colestático). Assim como outras doenças granulomatosas, a sarcoidose pode levar a um quadro de hipertensão portal ${ }^{4}$.

O diagnóstico da sarcoidose deve ser baseado na apresentação clínica e radiológica do paciente, devendo, porém, ser sempre confirmado por exame histológico. A biópsia deve mostrar granuloma, na ausência de agentes infecciosos (micobactérias ou fungos) ou de corpo estranho'.

É importante sempre descartar outras possíveis causas para as manifestações apresentadas. Para isso, deve-se usar o grande arsenal de testes imunológicos e de microbiologia disponíveis. Como anticorpo anticitoplasma de neutrófilo (ANCA) para granulomatose de Wegener e outras vasculites; anticorpo antimitocôndria para cirrose biliar primária; testes cutâneos, sorologias e cultura para fungos e micobactérias. No nosso meio, em que a tuberculose é uma doença bastante prevalente, seu diagnóstico deve ser sempre excluído na suspeita de sarcoidose ${ }^{1}$.

No caso descrito, a paciente apresentava doença pulmonar como mostra a tomografia de tórax, que revelou espessamento difuso de paredes brônquicas, podendo ser essa a causa da dispnéia apresentada. Embora tenha recebido inicialmente tratamento para tuberculose, não se sabe se houve confirmação do diagnóstico na ocasião. Em 2001, a pesquisa de bacilo álcool-ácido resistente no lavado brônquico foi negativa. Poderia ter sido realizada cultura dessa amostra e teste cutâneo (PPD) para ajudar a excluir o diagnóstico de tuberculose.

A paciente apresentava também linfonodomegalia intratorácica, axilar e, provavelmente, intra-abdominal (correspondendo a massa encontrada na tomografia de abdome), que, como relatado previamente, é bastante freqüente na sarcoidose.

Apresentava, ainda, doença hepática caracterizada por hepatomegalia, aumento de enzimas canaliculares (gama-GT e fosfatase alcalina), com pequena elevação de transaminases e função hepática provavelmente normal (dado pelo coagulograma). O exame de ultra-sonografia de abdome mostra sinais de hipertensão portal, que podem justificar a presença de ascite e edema de membros inferiores. Com a dosagem dos auto-anticorpos foram excluídas hepatopatias auto-imunes. Na avaliação da ascite, a presença de um nível baixo de adenosina deaminase ajuda a afastar o diagnóstico de tuberculose. Na biópsia hepática, a evidência de granulomas epitelióides com células gigantes e necrose focal, na ausência de outras etiologias, permitiu firmar o diagnóstico de sarcoidose.

O tratamento instituído - corticoterapia - é o preconizado para a sarcoidose ${ }^{5}$. É indicado para pacientes com acometimento pulmonar sintomático, com lesões de órgãos vitais (sistema nervoso central ou coração) ou acometimento grave de outros órgãos. Embora não se tenham dados de estudos controlados, outros agentes imunossupressores podem ser usados quando há algum impedimento ao uso de corticoesteróide ou na tentativa de redução de sua dose ${ }^{5}$.

A paciente em questão passou a apresentar sintomas de "cansaço" com esforços e edema, que, como visto, poderiam ser justificados pelo acometimento pulmonar e hepático da sarcoidose. No entanto, esses sintomas foram acompanhados de "aceleração" do coração (que pode corresponder às extra-sístoles evidenciadas no eletrocardiograma ou a outras taquiarritmias) e alterações nos exames iniciais: com evidência de cardiomegalia na radiografia de tórax e outras alterações eletrocardiográficas (distúrbios de condução), o que leva a pensar em cardiopatia associada.

Em virtude do histórico da paciente, a hipótese de envolvimento cardíaco pela sarcoidose deve ser considerada a principal hipótese diagnóstica, conforme será discutido adiante. Porém, não se deve esquecer de alguns diagnósticos diferenciais importantes.

Trata-se de uma paciente de 59 anos, obesa, dislipidêmica (colesterol total inicial de $364 \mathrm{mg} / \mathrm{dl}$ ), com glicemia de jejum alterada $(119 \mathrm{mg} / \mathrm{dl})$, e que, portanto, apresenta fatores de risco importantes para doença arterial coronariana, podendo ter desenvolvido assim miocardiopatia isquêmica. A realização de exames complementares para afastar esse diagnóstico torna-se essencial. No caso, foram realizados um ecocardiograma e uma ressonância cardíaca, que não sugerem essa etiologia.

Outro diagnóstico que deve ser lembrado é o de miocardiopatia chagásica. A paciente é natural de Minas Gerais, região endêmica para a doença. Apresentava no eletrocardiograma bloqueio de ramo direito associado a bloqueio da divisão ântero-superior do ramo esquerdo, alteração clássica na doença de Chagas, podendo ocorrer em até $30 \%$ dos pacientes ${ }^{6}$. Evoluiu com sintomas de insuficiência cardíaca e cardiomegalia, manifestações da forma cardíaca da doença. Seria, portanto, de extrema importância a realização de exame sorológico específico.

Devem ser lembradas ainda outras causas de miocardiopatia dilatada, como miocardite, hipertensão pulmonar (que, no caso, poderia ser secundária à pneumopatia existente) e a forma idiopática da doença. 
Para melhor avaliação do quadro, foi realizado um ecocardiograma que revelou ventrículo esquerdo de tamanho e função sistólica normais; espessamento de valvas mitral e aórtica, com leve insuficiência mitral; câmaras direitas e pericárdio normais.

Por meio desse exame é possível afastar cardiopatias que cursam com dilatação ventricular e disfunção sistólica predominante. Entretanto, devem ser lembradas outras cardiopatias que levam a disfunção diastólica. Entre elas estão as cardiomiopatias restritivas e as pericardiopatias constritivas. No caso em questão, as doenças do pericárdio podem ser afastadas pela ressonância magnética, que é um exame com grande acurácia para sua avaliação ${ }^{7}$.

A ressonância cardíaca da paciente revelou discreta disfunção do ventrículo esquerdo, com realce tardio dos ventrículos direito e esquerdo, compatível com cardiomiopatia de etiologia não-isquêmica.

As cardiomiopatias restritivas são distúrbios miocárdicos caracterizados por paredes ventriculares rígidas e nãocomplacentes de um ou ambos os ventrículos, mais comumente o esquerdo, impedindo o seu adequado enchimento ventricular e com conseqüente aumento da pressão diastólica final. Sua etiologia, geralmente, é de causa desconhecida e compreende a forma menos prevalente dentre as cardiomiopatias.

Essa entidade subdivide-se em três tipos: i) as infiltrativas miocárdicas, em que o miocárdio encontra-se infiltrado por uma substância anormal (amiloidose, sarcoidose, doença de Gaucher, hemocromatose, doença de Fabry); ii) as fibróticas miocárdicas, em que a fibrose se estende por todo o miocárdio (cardiomiopatia familiar, idiopática, secundária a radioterapia) e iii) as fibróticas endomiocárdicas, em que a fibrose acomete, especialmente, o endocárdio e o subendocárdio (endomiocardiofibrose, síndrome hipereosinofilica e metástases cardíacas de doenças tumorais) ${ }^{8}$.

Em relação às causas fibróticas endocárdicas, tem-se a síndrome hipereosinofílica ou doença de Löffler, caracterizada por uma eosinofilia maior que $1.500 / \mathrm{mm}^{3}$ por período maior que seis meses. Manifesta-se como miocardite com sintomas de insuficiência cardíaca congestiva de início subagudo, associada a febre, fadiga, perda de peso e rash cutâneo, ausentes no quadro clínico da paciente. Pode haver um quadro de mononeurite múltipla, encefalopatia e hepatoesplenomegalia, associados a arterite aguda e subseqüente formação de trombo no endocárdio, em cordas e valvas atrioventriculares, progredindo para fibrose. A doença afeta geralmente homens entre 30 e 40 anos, em países de clima temperado. Costuma ser progressiva, evoluindo com fibrose endomiocárdica com um padrão semelhante à endomiocardiofibrose tropical. Sem tratamento específico, a sobrevida é de apenas $25 \%$ em três anos ${ }^{9}$. Esse tipo de evolução não era apresentado pela paciente, o que torna improvável tal diagnóstico.

Outra causa presente no nosso meioéa endomiocardiofibrose, doença endêmica na África Equatorial, na Índia, na Colômbia e no Brasil, sem etiologia específica. As manifestações clínicas costumam aparecer na infância tardia, na adolescência ou na fase adulta precoce, diferentemente do apresentado pela paciente. A prevalência é igual entre os sexos, com predomínio na etnia negra. É uma doença de progressão variável, sendo a sobrevida nos estágios mais precoces de muitos anos; porém, em estágios avançados, de apenas $50 \%$ em dois anos. O aspecto do coração nessa doença é típico: a fibrose acomete a via de entrada ventricular, o ápice e o aparelho valvar atrioventricular, levando freqüentemente a regurgitação tricúspide e/ou mitral. A fibrose no ápice leva a sua obliteração ("cardiomiopatia obliterativa"), que algumas vezes pode ser decorrente de trombo apical. Na maioria dos casos, os dois ventrículos são acometidos. No quadro clínico predominam os sintomas e os sinais de congestão direita: turgência jugular, hepatomegalia, ascite, edema de membros inferiores. A congestão pulmonar leva a sintoma de dispnéia e ortopnéia. O diagnóstico é feito pelo aspecto morfológico característico na ressonância cardíaca e no ecocardiograma bidimensional, às vezes confirmado pela ventriculografia durante o cateterismo cardíaco $^{10}$, o que não foi encontrado na paciente.

Dentre as doenças infiltrativas do coração, devemos destacar a amiloidose, que acomete principalmente os rins (levando a síndrome nefrótica e insuficiência renal crônica), a pele, o subcutâneo, a língua, o sistema reticuloendotelial (levando a hepatoesplenomegalia) e o sistema nervoso periférico, sendo o comprometimento cardiovascular posterior ao envolvimento sistêmico. O diagnóstico é sugerido pelo comprometimento sistêmico e pelo aspecto ecocardiográfico (miocárdio "cintilante"). A confirmação pode ser feita pelo histopatológico do aspirado da gordura subcutânea abdominal. Nos casos duvidosos, pode ser realizada uma biópsia endomiocárdica. O prognóstico é ruim: a sobrevida média é de seis meses ${ }^{9}$. Essa etiologia se torna improvável para a paciente, pois, além de não apresentar o aspecto ecocardiográfico típico, apresentou longo tempo de evolução da doença, em torno de cinco anos, sem ter acometimento renal.

As outras doenças infiltrativas - hemocromatose, doença de Fabry, doença de Gaucher - são doenças de fundo genético que acometem, geralmente, pacientes em idades precoces e com história familiar. O acometimento sistêmico é característico, sendo a esplenomegalia comum à maioria dessas doenças infiltrativas de depósito, não encontrado na paciente, afastando tais possibilidades diagnósticas.

Deve-se destacar como hipótese diagnóstica principal o acometimento cardíaco pela sarcoidose. Esse ocorre em mais de 20\% dos casos; porém, apenas 5\% dos pacientes apresentam sintomas. A manifestação cardíaca pode ocorrer muitos anos após o diagnóstico inicial da doença. De qualquer forma, o aparecimento de sinais e sintomas cardiológicos em pacientes com sarcoidose exige uma investigação agressiva para o comprometimento cardíaco pela doença ${ }^{11}$.

Casuísticas de necropsia mostram que o miocárdio é mais freqüentemente envolvido; porém pode haver extensão do envolvimento para o pericárdio e o endocárdio. As áreas mais envolvidas são: a parede livre do ventrículo esquerdo, o septo interventricular e os músculos papilares ${ }^{12}$. O acometimento de músculo papilar, em geral, leva a disfunção valvar leve. Podem-se encontrar aneurismas no ventrículo esquerdo em até $10 \%$ dos pacientes com acometimento miocárdico.

A infiltração granulomatosa do sistema de condução e da parede ventricular pode levar a arritmias e defeitos de condução, sendo essa a manifestação mais comum 


\section{Correlação Anatomoclínica}

do acometimento cardíaco da doença. São observados variados graus de bloqueio atrioventricular, atraso de condução interventricular, bloqueio de ramo, extra-sístoles e taquicardias ventriculares. Arritmia é a principal causa de morte na sarcoidose cardíaca, seguido por insuficiência cardíaca progressiva secundária à infiltração miocárdica por granulomas ${ }^{11}$.

O diagnóstico de sarcoidose cardíaca é difícil, e muitas vezes o clínico deve iniciar o tratamento mesmo na ausência do diagnóstico histológico definitivo.

O ECG de repouso pode estar alterado em $20 \%$ a $30 \%$ dos pacientes com sarcoidose. Essas alterações são, em geral, inespecíficas, como alterações de segmento ST e onda T, presença de extra-sístoles e distúrbios de condução. A monitorização com Holter pode identificar taquiarritmias graves ou bloqueios cardíacos em pacientes assintomáticos, devendo ser sempre realizada. Qualquer alteração no eletrocardiograma ou no Holter requer maior avaliação com exames de imagem ${ }^{11}$.

O ecocardiograma detecta anormalidades em até 40\% dos pacientes com sarcoidose. Essas anormalidades incluem: hipocinesia ou discinesia focal ou global, aumento de câmaras, adelgaçamento da parede ventricular ou aneurismas, dilatação ventricular ou hipertrofia, fração de ejeção deprimida, disfunção diastólica, regurgitação valvar, derrame pericárdico. Alterações segmentares acometem preferencialmente as paredes anterior e apical ${ }^{11}$.

Os exames com radioisótopos são uma importante ferramenta diagnóstica. A cintilografia perfusional com uso do tálio201 parece superior ao ecocardiograma em mostrar alteração segmentar de contração de parede. As áreas de necrose ou fibrose não captam o fármaco; porém, na fase de estresse, pode ocorrer a regressão total ou parcial do defeito (fenômeno chamado de distribuição reversa, que se diferencia do padrão de alteração da doença arterial coronariana). Além disso, as áreas com defeito de perfusão na sarcoidose divergem do território de distribuição coronária. A cintilografia com gálio pode identificar áreas com processo inflamatório ativo. Alguns estudos sugerem que a combinação de defeito de perfusão com tálio e captação de gálio pode predizer a resposta a corticoesteróide.

Por ter uma resolução espacial superior, a ressonância magnética é considerada o melhor exame de imagem na avaliação da sarcoidose. Os infiltrados granulomatosos são vistos como zonas intramiocárdicas de sinal mais intenso, sendo mais evidente nas imagens em T2, em razão do edema associado à inflamação das lesões granulomatosas, apresentando realce com gadolíneo ${ }^{13}$.

A cineangiocoronariografia tem utilidade em pacientes com suspeita de sarcoidose cardíaca para descartar doença arterial coronariana. A biópsia endomiocárdica apresenta baixa sensibilidade (menor do que $25 \%$ ), devendo apenas ser realizada quando a doença multissistêmica não é evidente.

O prognóstico da sarcoidose cardíaca é variável, sendo os pacientes sintomáticos de maior risco. O tratamento com corticoesteróides parece ser eficaz e seu uso antes do desenvolvimento de disfunção sistólica está associado a um melhor desfecho clínico. A sobrevida em dez anos de pacientes com fração de ejeção maior que 50\% tratados com corticoesteróide é de $89 \%$, comparada a $27 \%$ de sobrevida nos pacientes com fração de ejeção inferior a 50\% tratados da mesma forma ${ }^{14}$. O marcapasso definitivo é indicado na presença de bloqueio atrioventricular completo ou outros graus de acometimento do sistema de condução. O uso de corticóide deve ser continuado de acordo com a tolerância e a necessidade clínica nos pacientes com marcapasso. É indicado o uso de cardiodesfibrilador implantável para pacientes com história de arritmias ventriculares graves.

Em relação ao caso, a paciente apresentava um quadro clínico e alterações de exames compatíveis com o diagnóstico de sarcoidose com envolvimento cardíaco. Na evolução, apresentou episódios de pré-síncope que foram atribuídos à hipotensão postural evidenciada em exame físico. Já nessa ocasião teria sido importante a avaliação de possível causa arritmogênica para esses episódios, em virtude de sua prevalência na evolução da sarcoidose cardíaca.

Após dois anos, a paciente retornou com novo quadro de pré-síncope, sendo, dessa vez, evidenciado bloqueio atrioventricular de segundo grau Mobitz II. Conforme recomendado pelas diretrizes internacionais, foi indicada a passagem de marcapasso provisório.

O ecocardiograma dessa internação mostrou discreto aumento de ventrículo esquerdo e aumento moderado de átrio esquerdo; algum grau de hipertensão pulmonar e discreto derrame pericárdico. Todas essas alterações eram compatíveis com a doença da paciente. A cintilografia cardíaca com gálio foi negativa para processo inflamatório cardíaco, mostrando que as manifestações atuais devem-se a lesão estrutural crônica do sistema de condução.

Após diversas tentativas de implante do marcapasso, a paciente evoluiu com quadro infeccioso, caracterizado por febre, leucocitose e sinais infecciosos em sítio de implante de eletrodo do marcapasso (hiperemia e presença de secreção purulenta); foram colhidas hemoculturas e deu-se início a antibioticoterapia adequada.

A paciente, no entanto, evoluiu com sinais de má-perfusão tecidual - rebaixamento de nível de consciência, piora de função renal - e, finalmente, hipotensão não-responsiva a volume, caracterizando um quadro de choque séptico. Apesar do tratamento adequado da infecção e do suporte hemodinâmico, a paciente evoluiu com disfunção de múltiplos órgãos, com perda progressiva da função cardiovascular, renal e hepática, evoluindo a óbito.

(Dr. Fábio de Moraes Medeiros, Dr. Luis Fernando B. C. Seguro)

\section{Hipótese diagnóstica}

Sarcoidose sistêmica com acometimento hepático e cardíaco e distúrbios de condução próprios da doença, apesar do tratamento com imunossupressores, necessitando de implante de marcapasso provisório; infecção pelo marcapasso, não-responsiva ao tratamento clínico; choque séptico e posterior disfunção de múltiplos órgãos.

(Dr. Fábio de Moraes Medeiros, Dr. Luis Fernando B. C. Seguro) 


\section{Necropsia}

À macroscopia, a necropsia revelou a presença de lesões miliares, esbranquiçadas, em torno de $2 \mathrm{~mm}$ cada, difusas e bilaterais nos pulmões (fig. 3). O coração estava envolvido em moderada quantidade de material fibrinóide e tinha seu peso levemente aumentado (394 g; normal na mulher até cerca de 300 g). Abertas, as câmaras cardíacas não apresentavam particularidades, exceto por leve dilatação do átrio esquerdo e discreta hipertrofia do ventrículo direito. No exame do miocárdio havia área de fibrose na parede lateral mediana do ventrículo esquerdo e, também no ventrículo direito, em sua parede póstero-septal apical, a qual estava justaposta ao local de implante do eletrodo do marca passo (fig. 4). Cortes para estudo do sistema de condução mostraram igualmente área de fibrose no topo do septo interventricular. No restante, as valvas atrioventriculares e ventriculoarteriais não apresentavam particularidades macroscópicas. O fígado tinha aspecto macronodular, com traves fibróticas, estrias vinhosas e colestase. Além disso, havia trombose aguda em organização da veia porta e suas ramificações intra-hepáticas (fig. 5). Trombose aguda foi observada ainda nas veias esplênica e pancreática. O baço estava aumento, pesando $526 \mathrm{~g}$ (normal em torno de 150 g), esclerocongestivo, com nódulos esparsos de fibrose e hemossiderina do tipo Gandy-Gamna. Havia trama vascular proeminente em terço distal do esôfago e fundo gástrico. O conteúdo do estômago era representado por cerca de dois litros de líquido hemorrágico misturado ao suco digestivo. Havia também espessamento da parede da

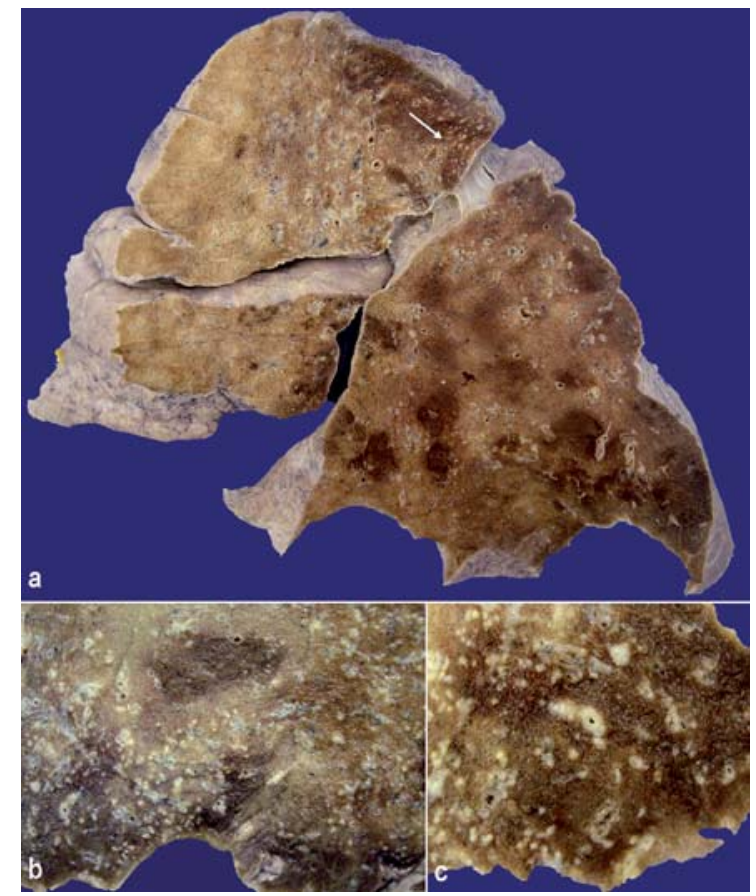

Fig. 3 - Macroscopia dos pulmões. Em (a), superfície de corte do pulmão direito com a presença de múltiplos nódulos miliares esbranquiçados, parte deles concentrados na base do lobo superior (seta). Em (b) e (c), visão aproximada dos nódulos miliares no pulmão esquerdo.

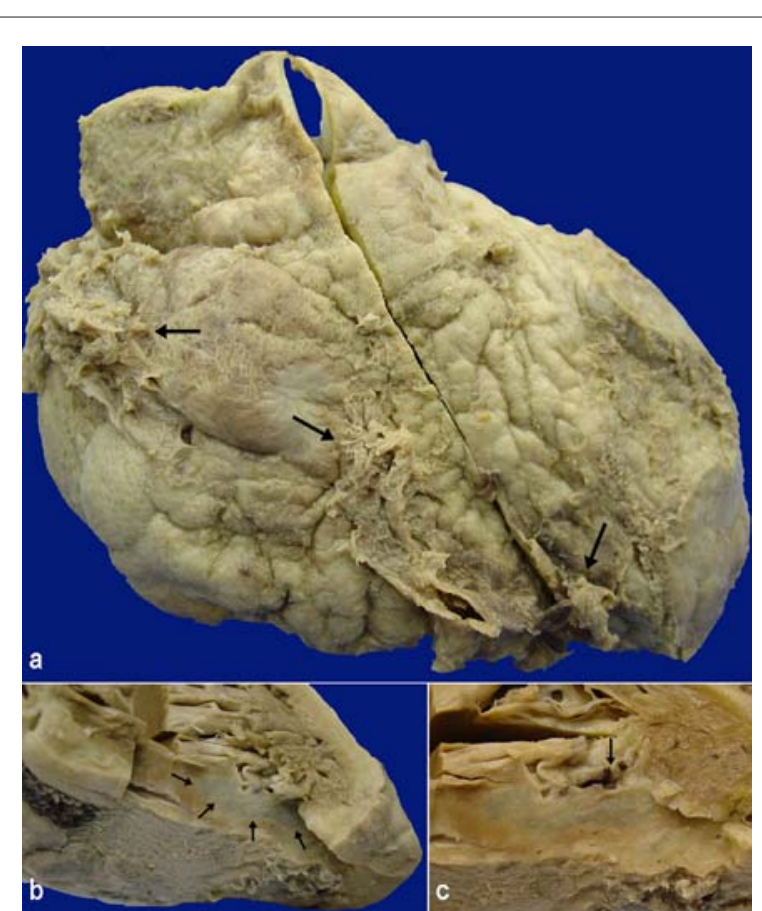

Fig. 4 - Aspecto frontal do coração com a superfície opacificada devido à cobertura de material fibrinóide, por vezes formando grumos (setas). Em (b), o coração está seccionado e as setas delimitam área de fibrose na parede póstero-apical do ventrículo direito. Em (c), o local onde estava preso o fio do marcapasso (seta), nas proximidades da área fibrótica mostrada em (b).

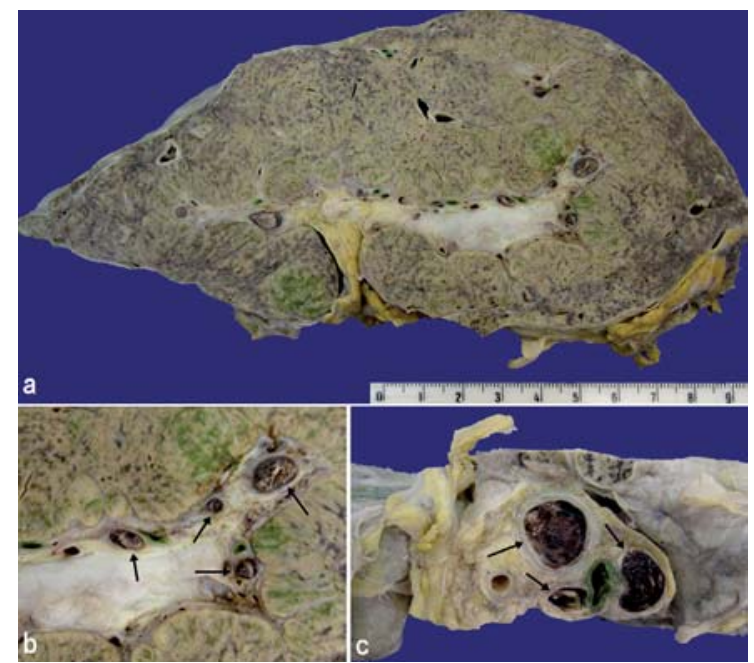

Fig. 5 - (a) Superfície de corte do fígado com aspecto macronodular, traves fibróticas, estrias vinhosas e colestase. Nas demais fotos, as setas indicam a trombose portal em ramos intra-hepáticos (b) e no hilo hepático (c).

veia cava inferior. Por último, ainda na macroscopia, foram observados: edema cerebral com herniação de amígdalas cerebelares (1.370 g; normal em torno de $1.200 \mathrm{~g}$ ); cistite aguda com áreas hemorrágicas, talvez associadas à sondagem; segmentos amolecidos de aspecto necrótico em alças 


\section{Correlação Anatomoclínica}

intestinais; colecistite crônica calculosa; cisto renal simples com 1,5 cm; e leiomioma uterino com $6,5 \mathrm{~cm}$.

Na histologia, foi observado comprometimento tecidual por múltiplos granulomas não-confluentes e não-caseosos, com padrão sarcoídico, em pulmões, baço, fígado (fig. 6) e coração, associados a extensas áreas de fibrose hialina, principalmente nestes dois últimos, correspondendo às áreas descritas na macroscopia. Nos pulmões, as lesões granulomatosas eram bronco e angiocêntricas. No coração, inclusive, a área descrita no topo do septo resultava em envolvimento do sistema de condução atrioventricular, ora com granulomas ora com fibrose, nas proximidades do nó atrioventricular, feixe de His e demais ramos (fig. 7). Observaram-se também inflamação e fibrose nas imediações do nó sinoatrial. Constatou-se ainda pericardite fibrinosa em organização. As colorações histoquímicas para agentes infecciosos, tais como bactérias, micobactérias e fungos, foram negativas nos tecidos com granuloma e também nos cortes do epicárdio. Para finalizar, a causa imediata da morte foi choque, com sua expressão morfológica observada em pulmões (edema intra-alveolar e congestão); fígado (áreas de necrose centrolobular); rins (necrose tubular aguda); sistema nervoso central (neurônios vermelhos); e intestinos (infartos da mucosa com trombose recente em vasos da submucosa).

(Dra. Jussara Bianchi Castelli)

\section{Diagnósticos anatomopatológicos}

Sarcoidose pulmonar, hepática, esplênica e cardíaca, com acometimento do sistema de condução; pericardite aguda fibrinosa em organização; cor pulmonale crônico leve; hipertensão portal com varizes de esôfago e fundo gástrico; hemorragia digestiva alta terminal; trombose de veias porta, esplênica e pancreática; choque séptico; colecistite crônica calculosa; cisto renal simples; leiomioma uterino.

(Dra. Jussara Bianchi Castelli)

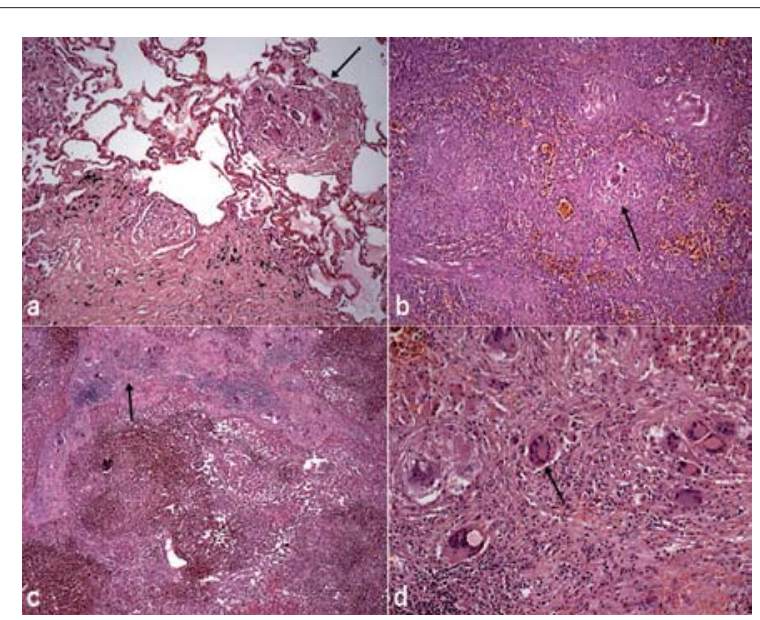

Fig. 6 - Histologia dos órgãos acometidos pela sarcoidose. Em (a), a seta aponta um dos granulomas clássicos presentes e, na base da foto, há área de fibrose. O baço (b) apresentava granulomas esparsos (seta). O fígado (c) estava bastante acometido por granulomas e fibrose (seta). Em (d), a seta indica uma das células gigantes do tipo Langhans presentes no granuloma (coloração de hematoxilina \& eosina)

\section{Discussão}

A sarcoidose é uma doença sistêmica que continua com causa desconhecida, apesar de inúmeros trabalhos até o momento. Nesses incluem-se várias pesquisas já empregando as recentes técnicas de biologia molecular com o intuito de verificar fatores genéticos e infecciosos ${ }^{15}$, com resultados díspares, sugerindo-se até que a causa talvez possa ser uma combinação desses e de outros fatores ${ }^{16}$.

Histologicamente é caracterizada pela presença de granulomas clássicos, não-confluentes e não-caseosos, quase sempre com células gigantes do tipo Langhans e de corpo estranho. Esse era o aspecto observado no caso, associado a áreas de fibrose, resultado da inflamação prévia e que tem caráter progressivo.

Os pulmões e os linfonodos hilares e mediastinais são os locais mais comuns de comprometimento pela sarcoidose. $\mathrm{O}$ coração é o terceiro órgão comumente afetado. Num estudo de necropsia, o coração estava acometido em 23 de 84 pacientes (27\%), e em um terço (8/23) esse envolvimento era clinicamente silencioso. Nos restantes $65 \%$, havia falência cardíaca e/ou defeitos de condução ou arritmia. Ainda nesse estudo, somente em 17\% (4/23) havia expressão macroscópica, que no restante foi vista unicamente ao microscópio, e em apenas um paciente havia comprometimento do sistema de condução, dos 20 casos nos quais esse foi examinado. Interessante a notar ainda é que,

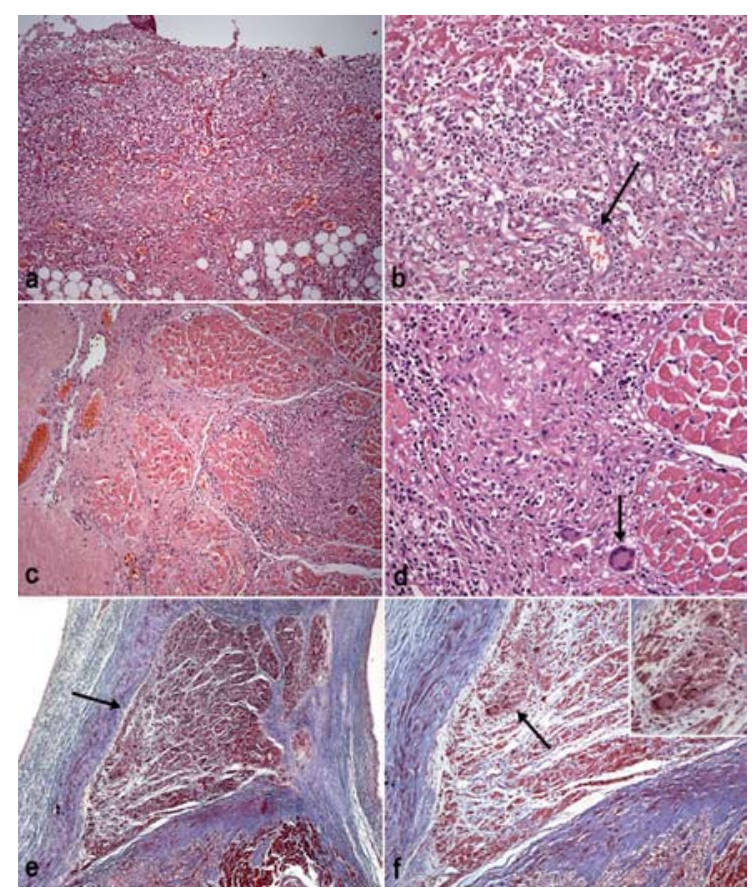

Fig. 7 - Histologia cardíaca. Em (a), corte do epicárdio com camada de fibrina permeada por células inflamatórias. No maior aumento (b) a presença dos vasos (seta) mostra organização do processo. Em (c), a histologia da região de fibrose na parede lateral do ventrículo esquerdo onde há múltiplos granulomas e deposição de colágeno. A foto (d) apresenta um destes granulomas com células gigantes (seta). Um dos setores do sistema de condução, o feixe de His, é demonstrado em (e) (seta), o qual apresenta envolvimento por granulomas. Este é mostrado em maior aumento em (f). [coloração: (a), (b), (c) e (d): hematoxilina \& eosina; (e) e (f): tricrômico de Masson]. 
nesse trabalho, dentre os 84 pacientes com sarcoidose, o grupo sem comprometimento cardíaco histológico apresentou uma sorte de arritmias, distúrbios de condução, falência cardíaca e morte súbita que puderam ser explicadas por outra causa que não a sarcoidose miocárdica, demonstrando a dificuldade diagnóstica, bem como sobreposição da manifestação clínica com outras doenças ${ }^{17}$.

No diagnóstico da sarcoidose a apresentação clínica é tão importante quanto a informação histopatológica. O diferencial histológico da sarcoidose deve ser feito com pneumonia de hipersensibilidade, micobacterioses, infecção fúngica e beriliose (pneumoconiose secundária à inalação de berílio, que era utilizado para a fabricação de lâmpadas). No coração ainda deve ser lembrada a miocardite de células gigantes ${ }^{18}$, que, apesar de não formar granulomas clássicos, poderia representar alguma dificuldade diagnóstica diferencial conforme as dimensões da biópsia. Doenças neoplásicas também podem causar reação granulomatosa, incluindo o linfoma, bem como neoplasias epiteliais de mama e de pulmão ${ }^{15}$. Assim sendo, o diagnóstico da sarcoidose é de exclusão, devendo seguir um algoritmo no qual a biópsia tem papel importante ${ }^{18}$.

Por fim, o comprometimento do parênquima pulmonar pela sarcoidose é descrito como uma das causas de hipertensão pulmonar ${ }^{19}$, esta última provavelmente presente neste caso. A sugestão disso está na leve hipertrofia do ventrículo direito, na discreta dilatação do átrio esquerdo, bem como na provável redução da complacência do leito vascular envolvido pelo processo granulomatoso e fibrótico observado na histologia pulmonar. Já a trombose de veia porta também já é descrita associada à fibrose hepática por sarcoidose e hipertensão portal ${ }^{20}$.

(Dra. Jussara Bianchi Castelli)

\section{Referências}

1. Newman LS, Rose CS, Maier LA. Medical progress: Sarcoidosis. N Engl J Med 1997; 336: 1224-34.

2. Winterbauer RH, Lammert J, Selland M, Wu R, Corley D, Springmeyer SC. Bronchoalveolar lavage cell populations in the diagnosis of sarcoidosis. Chest. 1993; 104: 352-61

3. Nishimura K, Itoh H, Kitaichi M, Nagai S, Izumi T. Pulmonary sarcoidosis: correlation of CT and histopathologic findings. Radiology. 1993; 189:105-9.

4. Friedman SL. Cirrhosis of the liver and its major sequelae. In: Cecil Textbook of Medicine 20 th ed. Philadelphia: Saunders Company; 1996. p. 788-95.

5. ATS/ERS Statement on Sarcoidosis. Am J Respir Crit Care Med. 1999; 160: 736-55.

6. Amorim DS, Marin Neto JA. Functional alterations of the autonomic nervous system in Chagas' heart disease. Sao Paulo Med J. 1995; 113 (2): 772-84.

7. Consenso SOCESP-SBC sobre ressonância magnética em cardiologia. Arq Bras Cardiol. 1995; 65: 451-7.

8. Barreto ACP, Dauar D. Cardiomiopatia restritiva. Rev Bras Med. 1986; 5 29-31.

9. Wynne J, Braunwald E. The Cardiomyopathies. In: Brawnwald's Heart Disease 7th ed. Phyladelphia: Saunders Company; 2005. p. 1659-96.

10. Salemi VCM, Mady C. Aspectos clínicos e fatores prognósticos em pacientes com endomiocardiofibrose. Rev Soc Cardiol Estado de São Paulo. 2003; 4: 509-15.

11. Deng JC, Baughman RP, Lynch JP. Cardiac involvement in sarcoidosis. Semin Respir Crit Care Med. 2002; 23: 513-28.

12. Roberts WC, McAllister HA Jr, Ferrans VJ. Sarcoidosis of the heart: clinicopathologic study of 35 necropsy patients (group 1) and review of 78 previously described necropsy patients (group 11). Am J Med. 1997; 63: 86-108.

13. Vignaux O, Dhote R, Duboc D, Blanche P, Dusser D, Weber S, Legmann P. Clinical significance of myocardial magnetic resonance abnormalities in patients with sarcoidosis. Chest. 2002; 122: 1895-901.

14. Yasaki Y, Isobe M, Hiroe M, Morimoto S, Hiramitsu S, Nakano T, etal. Prognostic determinants of long-term survival in Japanese patients with cardiac sarcoidosis treated with prednisone. Am J Cardiol. 2001; 88: 1006-10.

15. Baughman RP, Lower EE, du Bois RM. Sarcoidosis. Lancet. 2003; 361 : 1111-8.

16. Halushka MK, Yuh DD, Russell SD. Right ventricle-dominant cardiac sarcoidosis with sparing of the left ventricle. J Heart Lung Transplant. 2006; 25:479-82.

17. Silverman KJ, Hutchins GM, Bulkley BH. Cardiac sarcoid: a clinicopathologic study of 84 unselected patients with systemic sarcoidosis. Circulation. 1978; 58: 1204-11.

18. Okura Y, Dec GW, Hare JM, Kodama M, Berry GJ, Tazelaar HD, et al. A clinical and histopathologic comparison of cardiac sarcoidosis and idiopathic giant cell myocarditis. J Am Coll Cardiol. 2003; 15; 41: 322-9.

19. SrigleyJA, Pollanen MS. Sudden death with clinically undiagnosed pulmonary hypertension. J Clin Forensic Med. 2005; 12: 264-7.

20. Albernaz A, Rosa ACF, Machado MM, Barros N, Mota OM, Santana JB Fo, et al. Liver involvement in sarcoidosis: a case report. Radiol Bras. 2006; 39: 157-8. 